Kroneman, M.W., Essen, G.A. van, Tacken, M.A.J.B., Paget, W.J., Verheij, R.

Does a population survey provide reliable influenza vaccine uptake rates among high-risk groups?

Vaccine: 22, 2004, p. 2163-2170

\begin{tabular}{|l|l|}
\hline Postprint Version & 1.0 \\
\hline Journal website & $\underline{\text { http://www.sciencedirect.com }}$ \\
Pubmed link & $\begin{array}{l}\text { http://www.ncbi.nlm.nih.gov/entrez/query.fcgi?.cmd=Retrieve\&db=pubmed\&dop } \\
\text { t=Abstract\&list uids=15149773\&query hl=73\&itool=pubmed docsum }\end{array}$ \\
\hline DOI & 10.1016/j.vaccine.2003.11.049 \\
\hline
\end{tabular}

* Corresponding author. Tel.: +31-30-2729640; fax: +31-30-2729729. E-mail address:

m.kroneman@nivel.nl (M.W. Kroneman).

\title{
Does a population survey provide reliable influenza vaccine uptake rates among high-risk groups? A case- study of The Netherlands
}

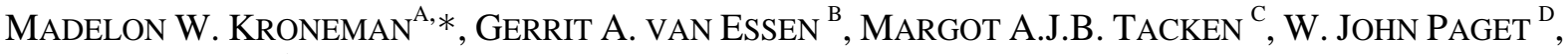 \\ ROBERT VERHEIJ ${ }^{\text {A }}$ \\ a Netherlands Institute of Health Services Research (NIVEL), P.O. Box 1568, 3500 BN Utrecht, The \\ Netherlands \\ b European Scientific Working Group on Influenza (ESWI), Julius Centre for Health Sciences and \\ Primary Care, University Medical Centre Utrecht, P.O. Box 85060, 3508 AB Utrecht, The \\ Netherlands \\ c LINH, Centre for Quality of Care Research (WOK), University Medical Centre Nijmegen, P.O. Box \\ 9101, 6500 HB Nijmegen, The Netherlands \\ d European Influenza Surveillance Scheme (EISS) Co-ordination Centre, NIVEL, P.O. Box 1568, 5300 \\ BN Utrecht, The Netherlands
}

\begin{abstract}
All European countries have recommendations for influenza vaccination among the elderly and chronically ill. However, only a few countries are able to provide data on influenza uptake among these groups. The aim of our study is to investigate whether a population survey is an effective method of obtaining vaccination uptake rates in the different risk groups and to find out what reasons people give as to why they have accepted or refused influenza vaccination and whether this varies among the risk groups. Amail questionnaire was sent out to households in The Netherlands, the response rate was 73\%. This resulted in data for 4037 individuals on influenza and influenza vaccination during the 2001-2002 influenza season. The uptake rates and size of different risk groups from the panel survey were comparable with other national representative sources (from the National Information Network of GPs (LINH) and Statistics Netherlands (CBS)). The main reason cited for undergoing vaccination was the existence of a chronic condition. The main reasons for refraining from vaccination were having enough resistance to flu and ignorance about the recommendations. In The Netherlands, the GP is the main administrator of influenza vaccines. We believe that population surveys may be useful for revealing influenza vaccination uptake rates for the groups at risk. When combined with questions about reasons for undergoing vaccination, the results may provide useful policy information and can be used to direct vaccination campaigns at under-vaccinated risk groups or to target the information campaign more effectively.
\end{abstract}

\section{INTRODUCTION}

All European countries have recommendations for influenza vaccination among the elderly and chronically ill [1]. However, only a few countries are able to provide data on vaccination uptake among these groups. About half of the European countries are able to report uptake rates for the elderly, and a few countries are able to do so for the other groups at risk [2]. However, insight into 
Kroneman, M.W., Essen, G.A. van, Tacken, M.A.J.B., Paget, W.J., Verheij, R.

Does a population survey provide reliable influenza vaccine uptake rates among high-risk groups?

Vaccine: 22, 2004, p. 2163-2170

vaccination uptake among different groups is essential for monitoring and evaluating national influenza vaccination campaigns, and for effectively directing information campaigns.

The aim of our study is to investigate the influenza uptake in the different risk groups. A second aim is to compare data from different sources, in this case panel data and data available from monitoring systems and to highlight the pro's and con's of both methods. A third aim is to find out what reasons people give as to why they have accepted or refused influenza vaccination and whether this varies among the risk groups.

We also look at the distribution channels of influenza vaccination, which may provide insight into the different health care providers that are involved in administering the vaccine.

For this study, the following research questions have been formulated: 1 . To what extent do various risk groups in The Netherlands receive influenza vaccination? 2. What reasons do people have for accepting or refraining from an influenza vaccination and does this vary among the risk groups? 3. What distribution channels are used and what is their relative share in total influenza vaccination in The Netherlands? Special attention will be given to the risk group due to disease that is under 65 years of age. There is no information available in the literature on vaccination uptake within this group.

\section{METHODS}

Data on vaccination uptake are available from various sources in The Netherlands.

Firstly, a mail questionnaire was sent to two existing panels that answer questions on health issues on a regular basis for Netherlands Institute of Health Services Research (NIVEL). The questionnaire contained nine questions about influenza vaccination (see Appendix A), as well as questions about the effects of influenza on sick leave used in another study. The data collection took place in May 2002 and concerned the vaccination campaign of 2001-2002. We asked the respondents to answer the questions for themselves and for all other members of their household. The respondents were asked to indicate for each household member whether they had an influenza vaccination, whether they belonged to a risk group, what reasons they had to accept or refrain from a vaccination, and who administered the vaccination.

\section{[ APPENDIX A ]}

For the definitions of the risk groups, we used the recommendations of the Dutch Health Council. A total of 2317 questionnaires were sent out to households in The Netherlands. Of these, 1692 (73\%) were completed and returned. This resulted in data for 4037 individuals on influenza and influenza vaccination during the 2001-2002 influenza season. In the results we will refer to this study as the 'panel'.

Differences in age distribution in the panel compared to the Dutch population are corrected for, by weighing the data of the respondents of 65 years and above and the category younger than 65 years with a factor calculated as follows: percentage in population divided by percentage in panel. The correction was performed as the survey had an over-representation of persons aged $>65$ (see below).

The second data source on vaccination uptake was the National Information Network of GPs (LINH). LINH provided data for the same season as the panel study [3]. The LINH data are based on 3,32,696 patients from 80 practices.

LINH data are representative of the Dutch population [4].

The LINH are derived from a registration project and therefore may be more reliable than panel data that are based on self-reported data about a past event. The LINH vaccination rate in the total population is comparable to the national vaccination rate based on data about the vaccine delivery (available from the National Program Influenza Prevention Foundation (SNPG)) [5].

The methods of LINH influenza monitoring have been described elsewhere [3,5]. In short, the routinely collected data were extracted from the computerised medical record systems of general practices with specially developed software.

The data were collected at the patient level. The recorded data included the patient's age, gender, type of health insurance (social health insurance or private health insurance), risk group, the influenza vaccination-indication tag, and whether vaccination had been given. 
Vaccination rates at the patient level were calculated for the total group of high-risk patients and for the different groups at risk separately [3]. Additional, for this study, vaccination rates were calculated for the risk group dichotomised for patients aged 65 years or older and the population under 65 years old. We will refer to the LINH data as 'population data'.

A third data source is a survey from Statistics Netherlands (CBS) for 2000 [6]. The CBS data are based on 5214 individuals. The survey is carried out on a continuous basis over the year. CBS data are representative of the Dutch population [7].

To answer the first research question, all three data sources will be used. Data for answering the second and third research question are only available from the panel.

\section{RESULTS}

\subsection{Representativeness}

Before comparing the datasources, we will investigate the representativeness of the panel data. The panel is representative of the Dutch population from the point of view of gender. There is a slight over-representation of the elderly (65 years or older) (see Table 1).

\section{[ TABLE 1 ]}

The distribution over the several categories of risk groups and non-risk groups is comparable between the panel and LINH (see Table 2).

\section{[ TABLE 2 ]}

In the panel data, there appears to be an under-representation of persons with diabetes mellitus (confidence interval for population data is 2.9-3.1\%). For heart disease, the confidence intervals of the panel study and the population data overlap (confidence interval for population data is 4.6-4.8\%). Within the risk groups younger than 65 years of age, an over-representation of patients with heart disease can be observed. For pulmonary diseases, the confidence intervals overlap (confidence interval for population data is 5.1-5.2\%). Roughly, the total group at risk exists for two-third out of elderly and one-third out of younger people, the latter group being included on the basis of their health status.

\subsection{Vaccination uptake and validity of the data}

Of all individuals in the panel, 24\% reported that they had been vaccinated against influenza (see Table 3). This is a higher uptake rate than reported via the LINH. Within the specific groups at risk, the situation is reversed. Compared to the data from Statistics Netherlands with uptake data from 2000, the uptake rate of the panel is higher. Within the specific groups at risk, the results are comparable. Within the population under 65 years of age, there appears to be an under-representation in the panel compared to the data from the LINH for the total group at risk and for those having pulmonary diseases in particular.

\section{[ TABLE 3 ]}

There appears to be variation among the risk groups in vaccination uptake. The uptake among the elderly is higher compared to those at risk under 65 years of age. The larger proportion non-vaccinated below 65 years of age can be found among those with pulmonary disease and those with less common diseases, like renal failure, low immunity due to disease or medical treatment and repeated staphylococcus.

\subsection{Reasons for participation in influenza vaccination}

The most important motivation for having been vaccinated against influenza was being aged 65 years or older and having a chronic condition (see Table 4).Within the risk groups, having a chronic condition was the most frequently mentioned motivating factor. Among the elderly, $81 \%$ cited age as a reason for being vaccinated, $15 \%$ mentioned only their chronic condition as reason. Among people 
with a heart disease, 52\% reported the disease as a reason, 24\% mentioned (also) having diseases from other risk groups and 29\% cited age. Among persons with pulmonary disease, this condition was reported by 70 and 15\% mentioned their age. For diabetics, this was comparable. For children, often no reason was reported, but where one was given, it was mainly pulmonary disease. There is no difference between the elderly and the younger population at risk due to their chronic condition in reasons for participation.

\section{[ TABLE 4 ]}

In Table 4, there are healthy adults (adults without a reported chronic condition in question 20, see Appendix A) who nevertheless cited having a chronic condition as a reason for vaccination (question 6, Appendix A). Other reasons for vaccination, reported by healthy adults, are: 'having another disease than the diseases for which vaccination is recommended', 'don't want to get the flu' and 'occupational risk'. An 'occupational risk' means, among other things, working in the healthcare sector or having a lot of contact with other people, as is the case among teachers.

\subsection{Reasons for not being vaccinated against influenza}

The most important reason for not being vaccinated is not qualifying for the vaccination (see Table 5). Of those who reported belonging to a high-risk group, almost a quarter of the non-vaccinated mentioned this as a reason for not being vaccinated. In all groups, about one third of the respondents thought they have enough resistance to flu. Other answers within the high-risk groups are: 'the physician considered vaccination unnecessary'; 'influenza is not severe' and; 'vaccination is redundant'.

\section{[ TABLE 5 ]}

Within the various high-risk groups, the reasons for not being vaccinated showed quite some diversity. The elderly are generally well aware of the fact that they qualify for a vaccination. Within the other risk groups this is less well known, since two out of five non-vaccinated persons from the high-risk groups due to disease report that they do not qualify for vaccination. Subsequently, most frequently cited reasons for not being vaccinated are: 'having enough resistance to flu', flu is not severe', and, 'vaccination is redundant'. The largest group to refrain from vaccination on principle was found among the elderly. A spontaneous answer given as a reason was 'bad experiences in the past'.

This is especially the case among the elderly (8\%), diabetics (8\%) and persons with pulmonary disease (6\%) (figures not shown in Table 5).

\subsection{Distribution channels}

In The Netherlands, the majority of vaccinations were administered by the GP. Within the group of adults without a chronic condition, a small proportion of respondents (6\%) indicated that the vaccination is administered by their company physician (this is a physician at the work place, that is involved in job related health issues). In the other groups, this seldom happens. Of the population at risk, 71\% received a personal invitation from the GP for the vaccination.

\section{CONCLUSION AND DISCUSSION}

In The Netherlands, there are several sources of information on influenza vaccination uptake. The most reliable is LINH, since these data are based on recording real vaccination uptake. However, survey data have similar results.

The panel-based survey of vaccination uptake seems to provide quite a reliable means of identifying uptake rates, although this requires some qualification. For the individual high-risk groups, the size and uptake rates were comparable with other national data sources. Only in the case of diabetes mellitus was a slight under-representation found.

The size of the panel sample (4037 individuals) contained sufficient numbers of persons in the four largest risk groups to be able to provide reliable uptake rates (the elderly, cardiovascular diseases, pulmonary diseases and diabetes mellitus). However, since both survey results (the panel and CBS 
Kroneman, M.W., Essen, G.A. van, Tacken, M.A.J.B., Paget, W.J., Verheij, R.

Does a population survey provide reliable influenza vaccine uptake rates among high-risk groups?

Vaccine: 22, 2004, p. 2163-2170

survey) revealed lower uptake rates for influenza vaccination compared to the LINH, survey data may lead to under-representation of vaccination uptake within the high-risk groups. Previous research supported the validity of self-reported uptake rates among the elderly [8-12], thus other factors may be the cause of this bias, for instance language problems among the non-native population and nonresponse of severely ill and very old persons. Panel data provide a relative reliable source of vaccination uptake.

The advantage of survey data is the relative cheap data collection compared to registration networks and the fact that other questions, for instance on reasons to comply with influenza vaccination can be added.

The main reason for undergoing vaccination is belonging to a high-risk group or, mainly for healthy adults, unwillingness to contract influenza. One group that stands out is the large percentage of healthy adults that report a chronic condition as a reason for undergoing vaccination. It is not clear why this is the case. It may be due to the design of the questionnaire: respondents may have omitted the last question.

Another possibility may be that these persons do not have a chronic condition confirmed by their physician, but persuaded their physician to administer a vaccination based on their perceived condition.

Two reasons were most frequently reported as a reason for refraining from influenza vaccination. Firstly, people believed they did not qualify for the vaccination. This is especially the case in the highrisk group under 65 years of age. Secondly, having enough resistance to flu was also an oftenmentioned reason. Among the elderly and those with pulmonary disease, 1 in 10 was of the opinion that influenza does not have serious effects. The elderly in particular refrain from vaccinations because of bad experiences in the past (like getting the flu despite vaccination and feeling bad after a vaccination). These reasons have also been found in other studies [13-21].

Two other reasons for not availing of vaccination — cost and distance-were not included in our study, because they are not applicable in The Netherlands. Vaccination in The Netherlands, including GP consultation, is free of charge for those at risk. In less densely populated countries, distance to the GP's surgery may play an important role, especially for the elderly [18]. Spontaneous answers by a relatively large number of persons ( $5 \%$ or more) indicated that they had had bad experiences with the vaccination in the past and that vaccination is redundant or unnecessary. These reasons may be included in the pre-printed answers if this survey is carried out in other European countries.

In The Netherlands, the GP plays a central role in administering the vaccine. In other countries, however, other distribution channels may play a role, such as public health organisations. In Denmark, for instance, about $69 \%$ of the elderly are vaccinated by their own GP and $24 \%$ are vaccinated at a vaccination clinic [22]. This raises the question as to whether distribution channels influence uptake rates.

In addition, in The Netherlands, a large proportion of the groups at risk receive a personal invitation for the vaccination.

In other countries, other recruitment methods may be used-which raises the question of the effect of different recruitment methods. Future research should address these questions.

One limitation of the panel is that belonging to a risk group is based on self-reporting by the population. This may lead to over-reporting as well as under-reporting. Another limitation is that the panel we used in The Netherlands specifically answers questions about health issues. This may result in bias because the participants tend to be more concerned with health issues. Furthermore, the fact that respondents were asked to complete the questionnaire for their family members as well may lead to bias, especially when asked for reasons for accepting or refraining from vaccination.

The advantages of this method were cost savings (with a limited number of questionnaires, data on more individuals is gathered). Finally, the timing of the survey - several months after the vaccination campaign - may result in mistakes in the answers. It would be better to plan the survey shortly after the end of the vaccination campaign (e.g. January).

For an international survey, a choice has to be made between a mail or telephone survey. A telephone survey is quicker and may receive a higher response. The survey should probably be integrated into an existing survey, as this would be more time- and cost effective. However, questions about household members complicate a telephone survey, and persons under the age of 16 cannot be questioned, due to 
Kroneman, M.W., Essen, G.A. van, Tacken, M.A.J.B., Paget, W.J., Verheij, R.

Does a population survey provide reliable influenza vaccine uptake rates among high-risk groups?

Vaccine: 22, 2004, p. 2163-2170

legal constraints. This will result in the omission of children and a higher number of respondents are needed.

Furthermore, the timing and format of the questionnaire are dependent on the organisation of the survey. However, within existing surveys, the number of respondents cannot be changed and this type of study requires relatively large numbers.

We believe that the results of the panel show that a relatively short questionnaire will give sufficient insight into the vaccination uptake among risk groups in a country. Although there may be some bias compared to the data from monitoring systems, survey data are easily obtainable at short notice.

Besides, a survey avoids the problem of the lack of data on the prevalence of chronic conditions, since respondents report their chronic conditions. We think that the lack of insight into uptake rates among risk groups in Europe could be solved in the short term by carrying out such a survey. In the longer term, a more reliable monitoring system could be developed, preferably in co-operation with European ScientificWorking Group on Influenza (ESWI) [18] and European Influenza Surveillance Scheme (EISS) [19]. Such information is essential in the discussion on pandemic planning.

In summary, we think that surveys may be useful for revealing influenza vaccination uptake rates for the groups at risk in a country. When combined with questions about reasons for undergoing vaccination, the results may provide useful policy information and can be used to direct vaccination campaigns at under-vaccinated risk groups or to target the information campaign more effectively.

\section{APPENDIX AND TABLES}

Questions in our questionnaire:

1 Please provide details of the members of your household.

Age for members 1-6

Gender for members 1-6

2 Did you or any of your household members receive a personal invitation from your GP for the influenza vaccination?

If yes, please indicate which household member(s) received a personal invitation.

3 Did you or any of your household members receive a vaccination against flu in October/November 2001 ?

If no, continue at question 7.

If yes: please indicate which household member(s) received an influenza vaccination.

4 Who advised you to have an influenza vaccination?
(a) GP
(b) Specialist
(c) Employer
(d) Public Health Association
(e) Own initiative
Other, namely ...

5 Who administered the vaccination to you or your household members?
(a) GP
(b) Company physician/occupational physician
(c) Public Health Association
(d) Other, namely ...

6 Can you indicate what was the reason for having an influenza vaccination?
(a) Heart disease (pain at the chest, heart rhythm disorders, heart attack or heart surgery)
(b) Lung disease (asthma, chronic bronchitis, pulmonary emphysema)
(c) Diabetes mellitus
(d) Renal disease (renal failure)
(e) Weakened immunity due to disease or medical treatment
(f) Repeated boils
(g) Age 65 years or over
(h) Other, namely ... 
Kroneman, M.W., Essen, G.A. van, Tacken, M.A.J.B., Paget, W.J., Verheij, R.

Does a population survey provide reliable influenza vaccine uptake rates among high-risk groups?

Vaccine: 22, 2004, p. 2163-2170

7 If you or one of your household members did not receive an influenza vaccination, please indicate why not.

(a) Does not qualify for influenza vaccination'

(b) Physician considered it unnecessary

(c) Enough resistance to flu

(d) Against vaccination on principle

(e) Influenza is not severe

(f) It slipped my mind

(g) Was unable to attend the vaccination times

(h) Other, namely. . .

20 Did a physician diagnose one of the following diseases in you or one of your family members?

(a) Heart disease (pain at the chest, heart rhythm disorders, heart attack or heart surgery)

(b) Lung disease (asthma, chronic bronchitis, pulmonary emphysema)

(c) Diabetes Mellitus

(d) Renal disease (renal failure)

(e) Weakened immunity due to disease or medical treatment

(f) Repeated boils

Questions 8 to 19 were not related to our study. Question 20 was deliberately placed at the end to avoid confusion with question 6 .

Table 1

Distribution of age and gender within the panel $(n=4037)$ compared to the national population ${ }^{\mathrm{a}}$

\begin{tabular}{lll}
\hline & Panel (\%) & Population (\%) \\
\hline Gender & & \\
$\quad$ Man & 48.7 & 49.5 \\
Woman & 51.3 & 50.5 \\
Age & & \\
$0-19$ & 22.1 & 24.4 \\
$20-64$ & 58.6 & 62.0 \\
65 and older & 19.3 & 13.6 \\
\hline
\end{tabular}

a The source of the population age and gender figures is the CBS year report 2001 [23].

Table 2

Distribution high-risk groups within the panel compared to the national population (LINH)

\begin{tabular}{|c|c|c|c|c|c|}
\hline & \multicolumn{4}{|l|}{ Panel } & \multirow{2}{*}{$\begin{array}{l}\text { Population } \\
(\%)\end{array}$} \\
\hline & Number & $\%$ & $\begin{array}{l}\text { Corrected for age } \\
\text { distribution }(\%)\end{array}$ & $\begin{array}{l}95 \% \text { age corrected } \\
\text { confidence interval }\end{array}$ & \\
\hline Total population at risk ${ }^{\mathrm{a}}(n=4037)$ from which ${ }^{\mathrm{b}}$ & 1163 & 28.8 & 23.6 & $22.3-27.9$ & 22.6 \\
\hline 65 years and older & 777 & 19.2 & 13.5 & $12.4-14.6$ & 12.7 \\
\hline Heart disease (all ages) & 176 & 4.4 & 4.0 & $3.4-4.6$ & 4.7 \\
\hline Pulmonary disease (all ages) & 242 & 6.0 & 6.0 & $5.3-6.7$ & 6.3 \\
\hline Diabetes mellitus (all ages) & 94 & 2.3 & 2.1 & $1.7-2.5$ & 3.0 \\
\hline Chronic renal failure (all ages) & 18 & 0.4 & 0.4 & $0.2-0.6$ & 0.3 \\
\hline Low immunity due to disease or medical treatment (all ages) & 102 & 2.5 & 2.6 & $2.1-3.1$ & Not available \\
\hline Repeated staphylococcus (all ages) & 12 & 0.3 & 0.3 & $0.1-0.5$ & 0.3 \\
\hline $\begin{array}{l}\text { Total population at risk under } 65 \text { years of age }{ }^{\mathrm{a}}(n=3260) \\
\text { from which }\end{array}$ & 386 & 11.8 & 11.8 & $10.7-12.9$ & 9.0 \\
\hline Heart disease & 101 & 3.1 & 3.1 & $2.5-3.7$ & 1.8 \\
\hline Pulmonary disease & 195 & 6.0 & 6.0 & $5.2-6.8$ & 5.1 \\
\hline Diabetes mellitus & 53 & 1.6 & 1.6 & $1.2-2.0$ & 1.6 \\
\hline Chronic renal failure & 11 & 0.3 & 0.3 & $0.1-0.5$ & 0.2 \\
\hline Low immunity due to disease or medical treatment & 87 & 2.7 & 2.6 & $2.1-3.1$ & Not available \\
\hline Repeated staphylococcus & 12 & 0.4 & 0.3 & $0.1-0.5$ & 0.2 \\
\hline
\end{tabular}

a The total population at risk for LINH includes all persons for whom the GP has indicated that vaccination is necessary and thus includes the group 'low immunity due to disease or medical treatment'.

${ }^{\mathrm{b}}$ People may belong to more than one risk group. 
Kroneman, M.W., Essen, G.A. van, Tacken, M.A.J.B., Paget, W.J., Verheij, R.

Does a population survey provide reliable influenza vaccine uptake rates among high-risk groups? Vaccine: 22, 2004, p. 2163-2170

Table 3

Influenza vaccination uptake rates for the different population groups

\begin{tabular}{|c|c|c|c|c|}
\hline & \multicolumn{2}{|c|}{ Panel } & \multirow{2}{*}{$\begin{array}{l}\text { National Information } \\
\text { Network of GPs } \\
\text { (LINH) }(\%)\end{array}$} & \multirow{2}{*}{$\begin{array}{l}\text { Statistics } \\
\text { Netherlands } \\
(\mathrm{CBS})^{\mathrm{a}}(\%)\end{array}$} \\
\hline & $\%$ & Confidence interval & & \\
\hline $\begin{array}{l}\text { Total population (panel corrected for age; } \\
\text { those not at risk included) }\end{array}$ & 24 & $22-25$ & 17 & 19 \\
\hline \multicolumn{5}{|l|}{ Groups at risk ${ }^{b}$} \\
\hline Total & 67 & $64-70$ & 75 & 67 \\
\hline Elderly $(>65)^{\mathrm{c}}$ & 74 & $71-77$ & 81 & $74^{\mathrm{d}}$ \\
\hline Cardiovascular diseases (all ages) & 77 & $71-84$ & 86 & 77 \\
\hline Pulmonary diseases (all ages) & 60 & $54-66$ & 73 & 54 \\
\hline Diabetes mellitus (all ages) & 73 & $64-82$ & 85 & 79 \\
\hline Population at risk under 65 years of age & 53 & $48-58$ & 70 & n.a. \\
\hline Cardiovascular diseases & 70 & $62-79$ & 79 & n.a. \\
\hline Pulmonary diseases & 54 & $47-60$ & 68 & n.a. \\
\hline Diabetes mellitus & 70 & $58-82$ & 79 & n.a. \\
\hline Other diseases (as reported in the panel) & 44 & $34-53$ & - & - \\
\hline
\end{tabular}

${ }^{a}$ Centraal Bureau voor de Statistiek (Statistics Netherlands), 2003 [6]

${ }^{\mathrm{b}}$ People may belong to more than one risk group.

' The group 'elderly' includes possible medical indications.

${ }^{\mathrm{d}}$ Data for $65-74,80$ years and older: $80.1 \%$.

Table 4

Reasons for participation in influenza vaccination (total vaccinated persons: 1066)

\begin{tabular}{|c|c|c|c|c|}
\hline Reason & $\begin{array}{l}\text { Total vaccinated } \\
\text { high-risk groups } \\
(n=779)^{\mathrm{a}}(\%)\end{array}$ & $\begin{array}{l}\text { Vaccinated elderly } \\
(n=573)^{\mathrm{a}}(\%)\end{array}$ & $\begin{array}{l}\text { Vaccinated high-risk } \\
\text { groups due to disease, age } \\
\text { under } 65(n=206)^{\mathrm{a}}(\%)\end{array}$ & $\begin{array}{l}\text { Vaccinated healthy } \\
\text { adults }^{\mathrm{a}, \mathrm{b}}(n=244)(\%)\end{array}$ \\
\hline Belonging to a risk group & 91 & 96 & 76 & 56 \\
\hline Other diseases & 1 & 0 & 3 & 9 \\
\hline Occupational risk & 0 & 0 & 1 & 4 \\
\hline Chronic illness Family member & 0 & 0 & 1 & 1 \\
\hline Does not want to get flu & 1 & 1 & 0 & 5 \\
\hline No answer & 8 & 3 & 21 & 23 \\
\hline
\end{tabular}

${ }^{a}$ More than one answer was allowed, therefore the individual percentages may add up to more than $100 \%$. The numbers $(n)$ are the vaccinated persons in our panel in the specific group.

${ }^{\mathrm{b}}$ Healthy adults are adults without a self-reported chronic condition (based on question 20, see Appendix A)

Table 5

Reasons for refraining from influenza vaccination (total non-vaccinated persons: $n=2971$ )

\begin{tabular}{|c|c|c|c|c|}
\hline Reason & $\begin{array}{l}\text { Total non-vaccinated } \\
\text { high-risk groups }{ }^{\mathrm{a}} \\
(n=383)(\%)\end{array}$ & $\begin{array}{l}\text { Non-vaccinated } \\
\text { elderly }^{\mathrm{a}} \\
(n=204)(\%)\end{array}$ & $\begin{array}{l}\text { Non-vaccinated high-risk } \\
\text { groups due to disease age } \\
\text { under } 65^{\text {a }}(n=179)(\%)\end{array}$ & $\begin{array}{l}\text { Non-vaccinated healthy } \\
\text { adults }^{\mathrm{a}, \mathrm{b}}(n=1888)(\%)\end{array}$ \\
\hline \multicolumn{5}{|l|}{ Pre-printed motivations } \\
\hline Does not qualify for vaccination & 23 & 9 & 38 & 56 \\
\hline Physician did not think it was necessary & 7 & 6 & 8 & 4 \\
\hline Enough resistance to the flu & 32 & 39 & 21 & 30 \\
\hline Against vaccination on principle & 5 & 7 & 2 & 2 \\
\hline Flu is not severe & 9 & 9 & 8 & 10 \\
\hline \multicolumn{5}{|l|}{ Spontaneously mentioned motivations } \\
\hline Bad experiences in the past & 6 & 8 & 4 & 1 \\
\hline Vaccination is redundant & 6 & 9 & 2 & 2 \\
\hline Other motivations & 14 & 12 & 16 & 5 \\
\hline No answer & 13 & 15 & 11 & 8 \\
\hline
\end{tabular}

a More than one answer was allowed, therefore the individual percentages may add to more than $100 \%$, only those reasons are presented that are cited by at least $5 \%$ of respondents in one of the groups. The numbers $(n)$ are the non-vaccinated persons in our panel in the specific group.

${ }^{\mathrm{b}}$ Healthy adults are adults without a self-reported chronic condition (based on question 20, see Appendix A). 